\title{
TRANSIENT DYNAMICS OF STREAK AND LONGITUDINAL VORTICES IN SHEAR FLOW WITH WAVE
}

\author{
MING ZHAO \\ Department of Engineering Mechanics, Dalian University of Technology \\ Dalian, PR China, \\ zhaoming@dlut.edu.cn \\ MOHAMED S. GHIDAOUI \\ Department of Civil and Environmental Engineering, \\ The Hong Kong University of Science and Technology, Hong Kong, PR China
}

\begin{abstract}
Langmuir circulations are alternating right and left helical vortices in the ocean having horizontal axes parallel to the wind. They are manifested by floating material on the ocean surface as streaks. The laboratory wind driven shear flow demonstrate streak feature with/without wave condition. When the wave is present the explanation of results with Craik-Leibovich instability mechanism, originally proposed for weak current shear in ocean, is not appropriate for some problem as in the laboratory the current shear is strong. The existing normal mode analysis based on the generalized Lagrangian mean formulation for wave with strong shear is not able to capture the fully dynamics of streak and longitudinal vortices. We extend Craik-Leibovich theory to strong shear in Eulerian framework but focusing on the fully perturbation evolution dynamics. Transient dynamics of perturbation is studied with an initial value problem. In this approach the effect of shear and wave and its interaction are made clear. The transient growth in the time period without wave is significant and is crucial to the initial preferred wave number observed in the experiments. The optimal longitudinal vortex form perturbation is searched and the preferred spanwise wave number predicted is well consistent with experimental data of wind shear and wave.
\end{abstract}

Key words: Langmuir circulation; wave and current interaction; stability analysis.

\section{Introduction}

Langmuir (1938) described his finding on August 7, 1927, when about 600 miles from New York on Atlantic crossing to England. He noticed that there were large quantities of floating seaweed, most of which was arranged in parallel lines with a somewhat irregular spacing ranging from 100 to 200 meters. These lines, parallel to the wind direction, are call streaks. His hypothesis is that the seaweed accumulated in streaks because of transverse surface currents converging toward the streaks. He also proposes that the action of the wind on the waters produce alternating right and left helical vortices in the water having horizontal axes parallel to the wind.

The widely accepted mechanism is due to Craik (1977) and carries the names CL-II mechanisms. The CL-II mechanism is due to flow instability. Span-wise variation in longitudinal perturbations generates vertical vorticity. The variability of the Stokes drift with depth tilts this vorticity in the streamwise direction. The descent and rise of water 
associated with streamwise vorticity generates vertical vorticity by tilting the horizontal (spanwise) vorticity of the current in the vertical direction. This process of positive feedback, where vertical vorticity interacts with stokes drift to generate streamwise vorticity, which in turn, interacts with the current to intensify vertical vorticity, leads to instability.

The verification of this mechanism, both under the field and laboratory conditions, is still far from complete. Faller and Caponi (1978) study the wind-driven LC in a shallow tank and find the dye bands on the bottom of tank due to the wind wave. In the experiment of Nepf and Monismith (1991) waves, which are generated mechanically, and current, which is generated by pump, are independent. Longitudinal vortices are found when wave exists. However, in their experiment, the circulations were strongly affected by the sidewalls of the tank used in the experiments. Melville et al. (1998) and Veron and Melville (2001) study the initial growth of the wind driven LC in a wave tank. The tank they used is sufficiently long, wide and deep to exclude end effects. They find initially there is only wind-driven current, after a certain time waves appear and then followed by Langmuir circulations.

Melville et al. (1998) try to explain their results with the stability diagram of Leibovich and Paolucci (1981), which is based on CL-II theory. Some other researchers go to different routes to explain the streaks observed on air-water interface without including wave. Handler et al. (2001) study the streak feature of air-water interface at wind speed $1-4 \mathrm{~m} / \mathrm{s}$, with it no surface wave is generated. The thermal structure observed clearly shows streaks on the surface. Tsai et al. (2001) use direct numerical simulation to study the turbulence of shear stress driven free surface flow. Surface wave is excluded in this case however the major surface features (streaks) observed in the laboratory and field experiments (Melville et al. 1998) is obtained in the simulation. These results of streak structure without wave indicates different phenomenon from Langmuir circulations.

On the other hand, CL-II mechanism is original proposed for Langmuir circulations in ocean, where the shear is weak compared to wave orbital velocity. The laboratory experiment of longitudinal vortex for shear and wave is not fully represented by the original CL-II since the current shear in this case is strong. Craik (1982) use generalized Lagrangian mean formulation to study stability for strong shear. Phillips (2005) uses similar approach to study the spacing of Langmuir circulations in strong shear and find good agreements with the data (Melville et al. 1998). Both Craik (1982) and Phillips (2005) use normal mode to study the growth of Langmuir circulation under strong shear. The growth rate obtained from normal mode approach represents the asymptotical growth of perturbation, which takes form of Langmuir circulation. However, the generation of streaky structures with or without wave in direct numerical simulations (Tsai et al. 2001) and in laboratory experiments (Melville et al. 1998, Handler et al. 2001) indicates a transient growth feature. Trefethen et al. (1993) mention that although the longitudinal vortices and streaks are persistent feature in shear flow they are not unstable mode predicted by eigen-value system. Bultler and Farrell (1992) find that the perturbation that can grow most transiently in viscous shear flows take the three-dimensional form very 
much close to streamwise vortex, although these perturbations asymptotically decays. The 2-D Squire mode can grow much smaller than those streamwise vortices. Schmid and Henningson (1994) find substantial transient growth followed by exponential decay in steady pipe flows. This transient growth is due to disturbances with no streamwise dependence and azimuthal wavenumber. Zhao et al. (2004 and 2007) extends the method of transient growth to unsteady pipe flows where normal mode analysis is not proper, and obtain quantitative results comparable to accelerating flows and decelerating flows in pipes. This approach is particularly useful when the time scale of the unsteadiness of the flow is either comparable to or smaller than the time scale of the growth of the instability. The method of transient growth provides the full time development of initially introduced perturbations in unsteady base flow condition investigates both bypass and exponential instabilities and examines the sensitivity of Langmuir circulations to the type of initial perturbations.

The aforementioned advantage suggests adopting transient stability analysis for the study of earlier stage of development of Langmuir circulations in strong current shear flow under wave, specifically those studied in Melville et al. (1998) and Veron and Melville (2001). The term "strong" is relative to wave representing that the current shear is of order 1 instead of order $\varepsilon^{s}, s \geq 1, \varepsilon=$ wave steepness.

\section{Stability Model}

\subsection{Base flow}

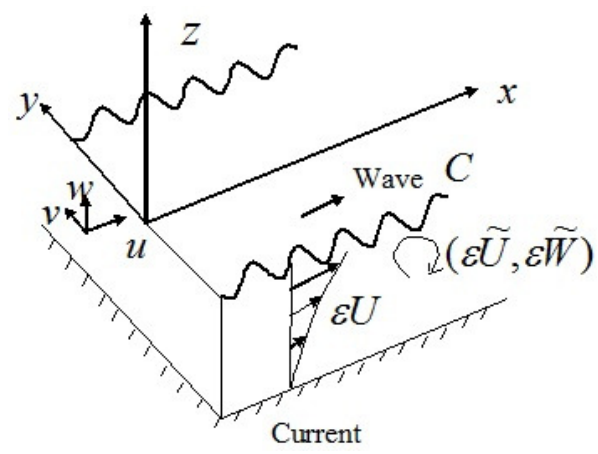

Fig. 1 Schematic plot of the flow problem

Fig. 1 depicts a wave-current base flow $(U, W)$, with $U$ the streamwise (longitudinal) component of velocity and $W$ the vertical component of velocity. The wave and current may be generated by wind or other type of forcing. The base flow expression is

$$
\begin{gathered}
U=U_{0}^{(1)}+\varepsilon\left(U_{1}^{(1)} e^{i \theta}+\text { c.c. }\right)+\cdots, \\
W=\varepsilon\left(W_{1}^{(1)} e^{i \theta}+\text { c.c. }\right)+\cdots \\
P=P_{0}^{(1)}+\varepsilon\left(P_{1}^{(1)} e^{i \theta}+\text { c.c. }\right)+\cdots
\end{gathered}
$$




$$
H=\varepsilon\left(H_{1}^{(1)} e^{i \theta}+\text { c.c. }\right)+\cdots,
$$

where $\varepsilon=a k$ is the wave steepness, $a$ is the characteristic wave amplitude and $k=2 \pi / \lambda$ is the characteristic longitudinal wave number, $\lambda$ is the characteristic longitudinal wave length, $(x, y, z)$ is longitudinal, span-wise and vertical coordinates normalized by $1 / k, U_{0}^{(1)}$ is current contribution to the base flow only in the longitudinal direction $(x),\left(\varepsilon U_{1}^{(1)}, \varepsilon W_{1}^{(1)}\right)$ is wave-induced component of the base flow along $x$ and $z$ direction, respectively. $\theta=x-t$ and $t$ is time, which is normalized by wave frequency $\omega$.

Primary current $U_{0}^{(1)}$ is normalized by wave phase velocity $C(C=\omega / k)$, and is of order 1 , and $U_{0}^{(1)}(z)-1 \neq 0$, otherwise critical layer exists in the flow. Shear $\partial U_{0}^{(1)} / \partial z \sim O(1)$ is assumed also. The wave is assumed independent of current, but of order $\varepsilon$, long-crested wave is assumed so that the wave is independent of spanwise direction and according to linear wave theory, wave components are

$$
U_{1}^{(1)}=\frac{A}{2} \frac{\cosh (k h+z)}{\sinh (k h)}, W_{1}^{(1)}=-i \frac{A}{2} \frac{\sinh (k h+z)}{\sinh (k h)}, P_{1}^{(1)}=\frac{A}{2} \frac{\cosh (k h+z)}{\sinh (k h)}, H_{1}^{(1)}=\frac{A}{2},
$$

where $A=A\left(x_{2}\right)$ is the wave amplitude which is attenuated slowly in $x$ direction $\left(x_{2}=\varepsilon^{2} x\right)$. In our calculation, the $x_{2}$ dependence is neglected. The secondary current due to wave is not included for simplicity, in this case since wave is of order $\varepsilon$, the secondary current is negligible in comparison to the primary current.

\subsection{Spanwise periodic disturbance to the current}

Since the basic current shear is of order 1 , the spanwide periodic disturbance in $x, y, z$ direction has different order for unstable mode (Craik 1982), where $u_{0}$ is $1 / \varepsilon$ greater than $v_{0}, w_{0}$. At the same time, the time dependence for the disturbance is related to slow variable $t_{1}=\varepsilon t$. Therefore, we assume disturbance in the form of

$$
\begin{gathered}
u^{\prime}=\gamma u_{0}\left(z, y, t_{1}\right)+\varepsilon \gamma\left(u_{1} e^{i \theta}+c . c\right)+\cdots, \\
v^{\prime}=\varepsilon \gamma v_{0}\left(z, y, t_{1}\right)+\varepsilon \gamma\left(v_{1} e^{i \theta}+c . c\right)+\cdots, \\
w^{\prime}=\varepsilon \gamma w_{0}\left(z, y, t_{1}\right)+\varepsilon \gamma\left(w_{1} e^{i \theta}+c . c\right)+\cdots,
\end{gathered}
$$

where $\gamma$ is infinitesimal and independent of $\varepsilon$. The subscript 0 represents time averaged perturbation (perturbed circulation), the subscript 1 represents the interaction of the mean perturbation and the wave. Our goal is to derive equations for the mean perturbation and study its development. The pressure and surface displacement are

$$
\begin{aligned}
& p^{\prime}=\varepsilon \gamma p_{0}\left(z, y, t_{1}\right)+\varepsilon \gamma\left(p_{1} e^{i \theta}+c . c\right)+\cdots, \\
& \eta^{\prime}=\varepsilon \gamma \eta_{0}\left(z, y, t_{1}\right)+\varepsilon \gamma\left(\eta_{1} e^{i \theta}+c . c\right)+\cdots
\end{aligned}
$$


where the pressure change and the surface displacement change due to the interaction between the basic current of $O(1)$ and perturbed velocity field of $O(\gamma)$ is assumed zero, which implicitly assume that the perturbation will not change the primary wave field. Therefore, the mean dynamic pressure (term with $p_{0}$ ) and the mean surface displacement (term with $\eta_{0}$ ) of perturbed circulation $(\sim O(\varepsilon \gamma))$ is smaller than the basic pressure and basic mean surface displacement $\left(\sim O(\varepsilon) \cdot p_{1}\right.$ and $\eta_{1}$ are those associated with the secondary wave. Therefore, the perturbed pressure and surface displacement of above form have same order of $v^{\prime}, w^{\prime}$.

Although generalized Lagrangian mean is used for strong shear case (Craik 1982, Phillips 2005), we follow the Eulerian approach by Huang and Mei (2006) to derive the governing equation and boundary condition. The detailed derivation is not listed here due to the length limitation. The continuity equation and momentum equations for mean perturbations are

$$
\begin{gathered}
\frac{\partial v_{0}}{\partial y}+\frac{\partial w_{0}}{\partial z}=0 \\
\frac{\partial u_{0}}{\partial t_{1}}+w_{0} \frac{\partial U_{0}^{(1)}}{\partial z}=\frac{v}{\varepsilon}\left(\frac{\partial^{2} u_{0}}{\partial y^{2}}+\frac{\partial^{2} u_{0}}{\partial z^{2}}\right) \\
\frac{\partial v_{0}}{\partial t_{1}}+\frac{\partial G}{\partial y}=\frac{v}{\varepsilon}\left(\frac{\partial^{2} v_{0}}{\partial y^{2}}+\frac{\partial^{2} v_{0}}{\partial z^{2}}\right) \\
\frac{\partial w_{0}}{\partial t_{1}}+\frac{\partial G}{\partial z}+u_{0} \frac{\partial U_{s}}{\partial z}=\frac{v}{\varepsilon}\left(\frac{\partial^{2} w_{0}}{\partial y^{2}}+\frac{\partial^{2} w_{0}}{\partial z^{2}}\right)
\end{gathered}
$$

where $v=v^{*} k / C$ is the dimensionless viscosity, $v^{*}$ is the kinematic viscosity of fluids. $G$ is a pressure like term and the gradient of Stokes drift is

$$
\frac{\partial U_{s}}{\partial z}=\frac{1}{1-U_{0}^{(1)}} \frac{A A^{*} \sinh 2(k h+z)}{\sinh ^{2}(k h)} .
$$

Boundary condition at the bottom and mean surface position are

$$
\begin{aligned}
& u_{0}=v_{0}=w_{0}=0 \quad \text { at } z=-k h, \\
& \frac{\partial u_{0}}{\partial z}=\frac{\partial v_{0}}{\partial z}=w_{0}=0 \text { at } z=0 .
\end{aligned}
$$

\section{Transient Stability Numerical Solutions}

Considering perturbation in the following form

$$
\left(u_{0}, v_{0}, w_{0}, G\right)\left(y, z, t_{2}, A\left(x_{2}\right)\right)=\operatorname{Re}\left\{\left(\hat{u}_{0}, i \hat{v}_{0}, \hat{w}_{0}, \hat{G}\right)\left(z, t_{2}, A\left(x_{2}\right)\right) e^{i k_{y} y}\right\} .
$$

Governing equations become 


$$
\begin{gathered}
-k_{y} \hat{v}_{0}+\frac{\partial \hat{w}_{0}}{\partial z}=0 \\
\frac{\partial \hat{u}_{0}}{\partial t_{1}}+\hat{w}_{0} \frac{\partial U_{0}^{(1)}}{\partial z}=\frac{v}{\varepsilon}\left(-k_{y}^{2}+\frac{\partial^{2}}{\partial z^{2}}\right) \hat{u}_{0} \\
\frac{\partial \hat{v}_{0}}{\partial t_{1}}+k_{y} \hat{G}=\frac{v}{\varepsilon}\left(-k_{y}^{2}+\frac{\partial^{2}}{\partial z^{2}}\right) \hat{v}_{0} \\
\frac{\partial \hat{w}_{0}}{\partial t_{1}}+\frac{\partial \hat{G}}{\partial z}+\hat{u}_{0} \frac{\partial U_{s}}{\partial z}=\frac{v}{\varepsilon}\left(-k_{y}{ }^{2}+\frac{\partial^{2}}{\partial z^{2}}\right) \hat{w}_{0} .
\end{gathered}
$$

Boundary conditions become

$$
\begin{gathered}
\hat{u}_{0}=\hat{v}_{0}=\hat{w}_{0}=0 \quad \text { at } z=-k h, \\
\frac{\partial \hat{u}_{0}}{\partial z}=\frac{\partial \hat{v}_{0}}{\partial z}=\hat{w}_{0}=0 \quad \text { at } z=0 .
\end{gathered}
$$

In this way, no exponential growth with respect to time is assumed. The growth can be transient.

The method of transient growth provides the full time development of initially introduced perturbations in unsteady base flow condition $\left(U_{0}^{(1)}\right.$ and $U_{s}$ can be time dependent), investigates both bypass and exponential instabilities and examines the sensitivity of Langmuir circulations to different types of initial perturbations. Three types of initial conditions are used. Type 1 assigns a random series to $\hat{u}(z, 0), \hat{w}(z, 0)$ and sets $\hat{v}(z, 0)=\hat{G}(z, 0)=0$. In order for this random field to satisfy continuity, an initial value problem comprising the random field with no forcing $\left(\hat{w}_{0} \partial U_{0}^{(1)} / \partial z=0\right.$, $\left.\hat{u}_{0} \partial U_{s} / \partial z=0\right)$ is solved. The resulting solution is a mass conserving random function, which is used as initial condition.

Type 2 initial condition assigns the asymptotic solution of the transient stability analysis, which is the eigenvector for the most unstable mode, to $\hat{u}(z, 0), \hat{w}(z, 0)$, $\hat{v}(z, 0)$ and $\hat{G}(z, 0)$. Type 3 is optimal in the sense that the $\hat{u}(z, 0), \hat{w}(z, 0)$, $\hat{v}(z, 0)$ and $\hat{G}(z, 0)$ for which maximize growth rate are chosen. Such optimal growth is not likely to occur in nature, but provides an upper bound for growth rate of Langmuir circulations.

The numerical solution of Zhao et al. $(2004,2007)$ is used, where Adams-Bashforth scheme is used for the convective terms and the Crank-Nicolson scheme is used for the viscous terms. The pressure-like term $(\mathrm{G})$ and continuity equation are solved implicitly. A collocation method based on Chebyshev polynomials is used for discretization in $z$ direction. The validation of the code for strong shear current is not applicable since no same formulation in the literature is available. Therefore, we use the code for weak shear current to validate the numerical solution of Huang and Mei (2006) (Zhao et al. 2010) gives the detailed validation) and assume the strong shear current code is justified since 
the difference between those two current shear formulations is trivial. The difference lies in three aspects. First, the time derivative is respect to $t_{1}=\varepsilon t$ instead of $t_{2}=\varepsilon^{2} t$. Second, the term representing Stokes drift change to Eq. (15), the denominator changes from 1 for the weak shear to $1-U_{0}^{(1)}$ for strong shear. Third, for strong current shear, the boundary shear stress is high order small term while the weak current shear the boundary surface shear stress is important.

A natural norm for investigating the time evolution of perturbation strength is the $L_{2}$ norm of $\left(u_{0}, v_{0}, w_{0}\right)$, denoted by

$$
E_{u_{0}}=\int_{-k h+z_{b}}^{0} \hat{u}_{0}^{2} d z, \quad E_{v_{0}}=\varepsilon^{2} \int_{-k h+z_{b}}^{0} \hat{v}_{0}^{2} d z, \quad E_{w_{0}}=\varepsilon^{2} \int_{-k h+z_{b}}^{0} \hat{w}_{0}^{2} d z,
$$

since $u_{0}$ is $1 / \varepsilon$ greater than $v_{0}$ and $w_{0}$, the above normal is at different order. The total energy for the perturbation is $E=\left(E_{u_{0}}+E_{v_{0}}+E_{w_{0}}\right)$.

\section{Results and Discussion}

Melville et al. (1998) carried out laboratory measurements of the generation and evolution of Langmuir circulations. A shear layer is generated by a linearly accelerated wind starting from rest above a quiescent water surface. Langmuir circulations are observed shortly after the initial growth of the wind generated wave. Comparing experiments with theory, Melville et al. (1998) found that the most unstable wave number inferred from the experiment is approximately twice that predicted from the normal mode theory. Melville et al. (1998) postulated that the discrepancy is likely due to (i) the current strength is of the order of the wave-phase speed $(C)$ in the experiment, but of the order of $\varepsilon^{2} C$ in the theory and (ii) the flow is unsteady in the experiment, but assumed as quasi-steady in the normal mode theory.

\subsection{Base flow and Stokes drift}

The wind-induced velocity current (Veron and Melville 2001) is

$$
U_{0}^{(1)}=\frac{U_{0}^{*}\left(t_{1}\right)}{C}\left[\left(1+2 \eta^{2}\right) \operatorname{erfc}(\eta)-\frac{2}{\sqrt{\pi}} \exp \left(-\eta^{2}\right)\right],
$$

where $\eta=-z^{*} / 2 \sqrt{v^{*} t^{*}}=-z / 2 \sqrt{v t_{1} / \varepsilon}, U_{0}^{*}$ is dimensional surface velocity. Therefore, the current shear is

$$
\frac{\partial U_{0}^{(1)}}{\partial z}=A \alpha\left[\exp \left(-\eta^{2}\right)-\sqrt{\pi} \eta \operatorname{erfc}(\eta)\right]
$$

where $A=\sqrt{t_{1} / T_{0}}$ when $t_{1}<T_{0} ; A=1$, when $t_{1} \geq T_{0} . T_{0}=T_{0}^{*} \omega \varepsilon$ is dimensionless time needed for the wind to reach its final speed, $T_{0}^{*}$ is dimensional time for the wind to reach its final speed, and 


$$
\alpha=\frac{2 U_{0 f}^{*}}{\sqrt{T_{0}^{*} \pi v^{*}}} \frac{1}{\omega},
$$

$U_{0 f}^{*}$ is dimensional final surface velocity.

The time-dependent shear of the developing-Stokes drift is

$$
\frac{\partial U_{s}\left(t_{1}, z\right)}{\partial z}=B\left(t_{1}\right) \frac{1}{1-U_{0}^{(1)}} \frac{\sinh 2(k h+z)}{\sinh ^{2}(k h)},
$$

where

$$
\left\{\begin{array}{l}
B=0 \quad \text { when } t_{1}<T_{1} \\
B=\left(\exp \left(\frac{t_{1}-T_{1}}{T_{2}-T_{1}}\right)-1\right) /(\exp (1)-1) \quad \text { when } T_{1}<t_{1}<T_{2} \\
B=1 \text { when } t_{1}>T_{2}
\end{array}\right.
$$

and $T_{1}$ and $T_{2}$ are the starting and ending times for the wave to grow exponentially, respectively. $T_{2}$ is also the perception time of Langmuir circulations, note both $T_{1}$ and $T_{2}$ are normalized by $\omega \varepsilon$, as the dimensionless time $t_{1}$ is used.

From the experimental data, the preferred wave number is $k_{y}=0.68$, wave slope is $\varepsilon=0.042, k h=144, U_{0 f}^{*}=0.124 \mathrm{~m} / \mathrm{s}, C=0.298 \mathrm{~m} / \mathrm{s}, \omega=36.7 \mathrm{~s}^{-1}, T_{0}^{*}=20 \mathrm{~s} . T_{0}=31.1$, $T_{1}=65.4, T_{2}=80.9$. The calculated $\alpha=0.853$ is $O(1)$, which is consistent with the scaling that the shear is strong (i.e., of order 1). The maximum current takes place at the surface and the value is $U_{0}^{(1)}=U_{0 f}^{*} / C=0.42$ is of order 1 , and $U_{0}^{(1)}(z)-1 \neq 0$. The assumption that there is no critical layer is validated.

\subsection{Initial condition and energy growth}

Unlike the normal mode approach, where the asymptotic behavior is searched, the initial value problem solves the full dynamics of initially introduced perturbation. The initial stage of perturbation dynamics depends on the initial conditions. We firstly apply random initial condition.
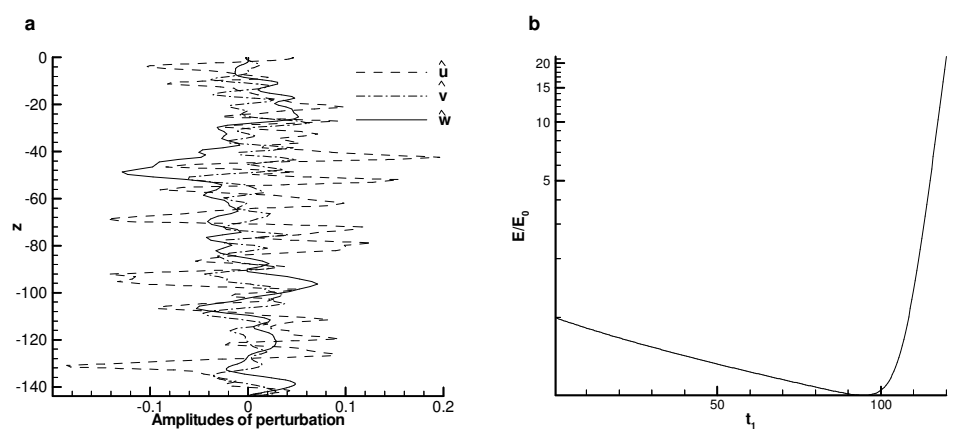

Fig. 2 (a) Amplitudes of one random initial perturbations as a function of water depth and (b) Energy growth for the random initial condition $\left(k_{y}=0.5\right)$ 
Fig. 2 plots the amplitudes of a random initial perturbation and its energy growth. The energy of the perturbation initially decays, and after $t_{1}=100$, it grows exponentially. This is because the current random initial perturbation includes many modes, all of them decays initially. The reason for decay is before $t_{1}=T_{1}=65.4$, there is no wave, the feedback of CL-II mechanism is missing, there is no instability. After $t_{1}=T_{1}$, wave sets in, the instability due to CL-II mechanism takes effect. Some of the modes begin to grow, however, other modes continue to decay and their amplitudes are still significant. With $t_{1}$ increases further, the amplitude of wave increases, the growth rate of instability increases. The energy percentage of decaying modes decreases. After $t_{1}=100$, the growth of the unstable modes dominants the decay of stable modes. The total energy begins to grow. After $t_{1}=110$, the energy grows approximate exponential. This is because that the base current shear due to diffusion changes slowly after $T_{0}$, and the gradient of Stokes drift is fixed after $T_{2}$, the forcing of the instability therefore is almost unchanged. For the current simulation period $t_{1}=120$, the perturbation energy grows significant. The final distribution of perturbation is the approximate eigenvector of the final system with almost constant current shear and constant gradient of Stokes drift.

Then we use the approximate eigenvector as initial perturbation and study its energy growth. The perturbation amplitude and its energy growth are plotted in Fig. 3.
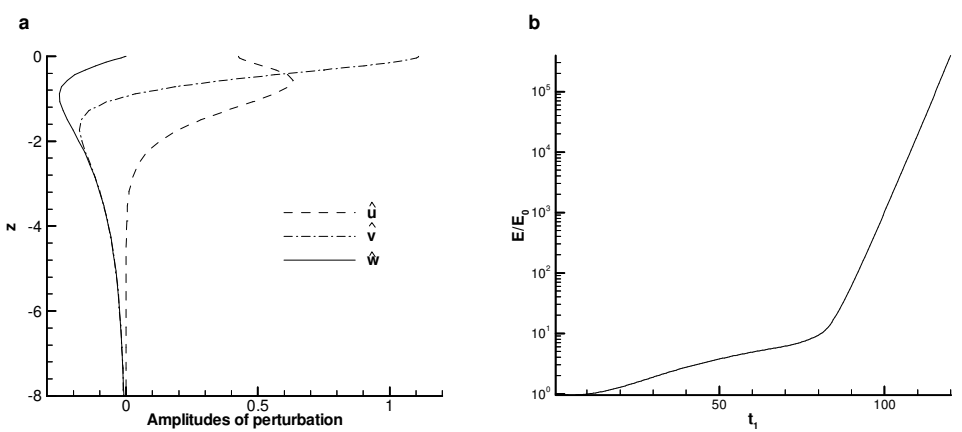

Fig. 3 (a) Amplitudes of approximate eigenvector and (b) Energy growth for the eigenvector initial condition

Although there is no wave before $t_{1}=T_{1}=65.4$, which means the CL-II instability is missing, there is slow growth of energy, this is due to the lift-up effect of the $\hat{w}_{0} \partial U_{0}^{(1)} / \partial z$ term, or transient growth. With this initial condition, at $t_{1}=T_{2}=80.9$, the energy already grow 10 times.

The energy growth rate is defined as following and plotted in Fig. 4.

$$
\sigma=\varepsilon \sigma_{1}=\varepsilon \frac{\partial \ln E}{\partial t_{1}} .
$$




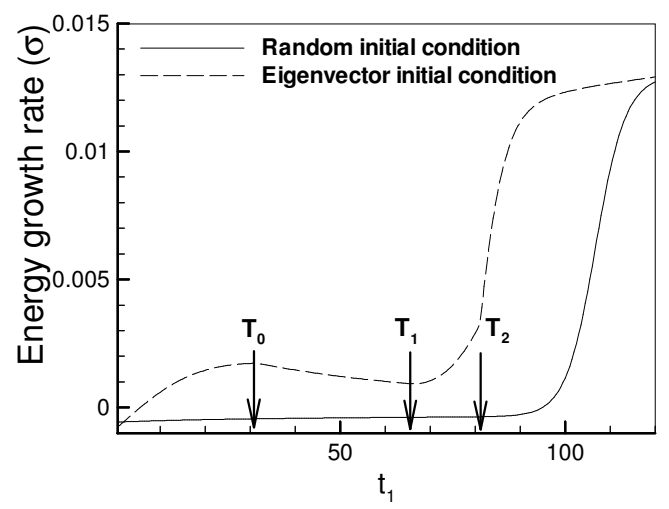

Fig. 4 Energy growth rate of perturbations with respect to time for different initial perturbations

The first arrow in the figure indicates the time when the surface velocity approaches its maximum value. The second and third arrows indicate the time period for the wave to growth (in terms of the amplitude of gradient of Stokes drift). It is shown that for the random initial perturbation case when the basic wave is weak, before $t_{1}=T_{1}=65.4$, the growth rate is negative, which means the initial perturbation is decaying. Only after $t_{1}=100$ when the basic wave approach a certain strength, and the growth modes dominants, the perturbation growth rate becomes positive and large, the perturbation growth fast and at the end of simulation time $t_{1}=120$. For the eigenvector perturbation case it demonstrates a small transient growth rate around $t_{1}=T_{0}=31.1$ and large growth rate after $t_{1}=T_{2}=80.9$. The current paper use 2-D wave and base shear as base flow for longitudinal perturbation development simulation. In fact, the 2-D wave used in the simulation is destroyed by the Langmuir circulations after that time $t_{1}=T_{2}$ as shown in the experiment. This illustrates the important of the 2-D wave for the initial growth of 3-D perturbation (Langmuir circulations).

The small transient growth rate associated with eigenvector initial perturbation gives some hint for the importance of transient growth. As the transient growth is highly initial condition dependent, an optimal initial perturbation is naturally needed to search.

\subsection{Optimal initial perturbation and preferred span-wise wave number}

The optimal initial perturbation and how it is related to the preferred span-wise wave number is discussed as follows. Although we can use a general optimization tool to find the initial perturbation that obtain largest growth at time $T_{2}$ as what has been done in Zhao et al. (2007), it is expected to be too time consuming. In this paper we use more specific approach for this particular problem. From Eq. (20), $\hat{w}_{0}$ lifts up the velocity gradient $\partial U_{0}^{(1)} / \partial z$ to produce $\hat{u}_{0}$. When there is no wave (Stokes drift gradient), the feedback mechanism of CL-II is missing, $\hat{w}_{0}$ itself is decaying due to eddy viscosity, this will weaken the lift up effect for $\hat{u}_{0}$. For larger span-wise wave number $\left(k_{y}\right)$ perturbation, it decays faster with growth rate $-d\left(k_{y}\right)=-k_{y}{ }^{2} v / \varepsilon$. However, when the wave exists, the feedback mechanism of CL-II sets in, the interaction of $\hat{u}_{0}$ and $\hat{w}_{0}$ 
creates growth of $\hat{u}_{0}$ and $\hat{w}_{0}\left(g\left(k_{y}\right)\right)$. It is found that the growth rate increase with span-wise wave number. Therefore, there exists a wave number $k_{y}$ that will make the initial perturbation obtain largest growth at time $T_{2}$. This wave number is defined as critical wave number. To be fair, the initial perturbation should be the optimal perturbation that gives largest growth for the particular wave number $k_{y}$. Obviously the optimal initial perturbation has $\hat{u}_{0}=0$, since $\hat{u}_{0}$ has no effect when the wave is absent. As the base current shear rate has maximum at surface and decays with depth (Fig. 3), $\hat{w}_{0}$ should be as large as possible near the surface, however, boundary condition requires $\hat{w}_{0}=0$ at $z=0$, and the final eigenvector perturbation mode (Fig. 3a) suggests that the perturbation has the following approximate form

$$
\hat{w}_{0}(z)=\frac{2}{\lambda^{2}} z \exp \left(-\left(\frac{z}{\lambda}\right)^{2}\right),
$$

which is Weibull distribution function with shape parameter of 2 . The corresponding $\hat{v}_{0}$ can be obtained with continuity equation (16). The only parameter $\lambda$ is a scale parameter, which shows where the peak is and the relative magnitude of the peak. Fig. 5 plots $\hat{w}_{0}$ for different $\lambda$.

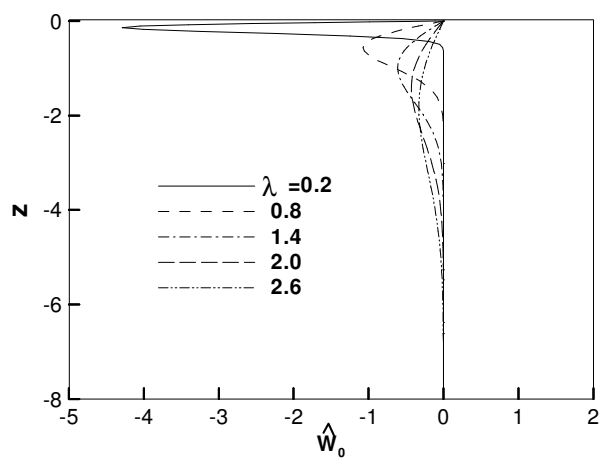

Fig. 5 Vertical initial perturbation for different scale parameter

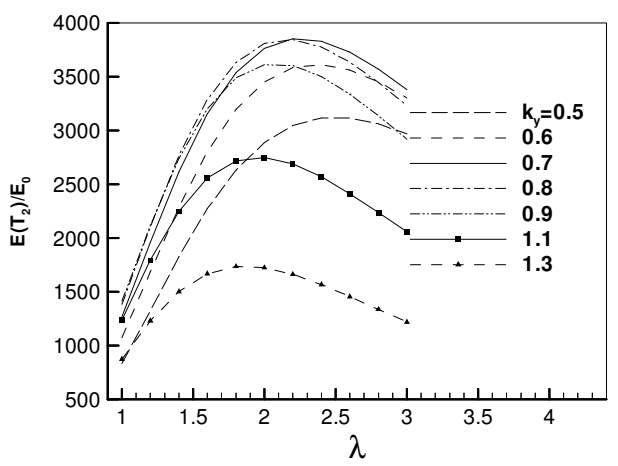

Fig. 6 Energy growth for different $\lambda$ and $k_{y}$ for final wind speed of $3 \mathrm{~m} / \mathrm{s}$ case 
For a certain wave number $k_{y}$, we can vary $\lambda$ to get different initial conditions. The energy growth at time $t_{1}=T_{2}$ can be calculated. The results are shown in Fig. 6 for final wind speed of $3 \mathrm{~m} / \mathrm{s}$ case. It is found that there is a maximum energy growth among those produced by different parameter $\lambda$ corresponding to a fixed $k_{y}$. For example, for $k_{y}=1.1$, the maximum growth is about 2750 at $\lambda=2.0$. For different wave number $k_{y}$, the maximum growth is different. The maximum growth (3850) among those maximums is obtained when the wave number is $k_{y}=0.7$ with the corresponding parameter $\lambda=2.2$. Therefore, according to the present definition, the critical wave number is $k_{y}=0.7$, that is the maximum value for $k_{y}=0.7$ is biggest among other maximum values. This result agrees with the experiment $k_{y}=0.68 \pm 0.2$ very well.

Fig. 7 plots the maximum energy growth for different $k_{y}$ and for different final wind speeds. The critical wave number for final wind speed $4 \mathrm{~m} / \mathrm{s}$ is $k_{y}=0.9$ also agrees with the experiment $k_{y}=0.71 \pm 0.36$. The corresponding parameter is $\lambda=2.4$ (from calculation, not shown here). The critical wave number for final wind speed $5 \mathrm{~m} / \mathrm{s}$ is $m=0.7$ also agrees with the experiment $k_{y}=0.72 \pm 0.23$. The corresponding parameter is $\lambda=2.8$.

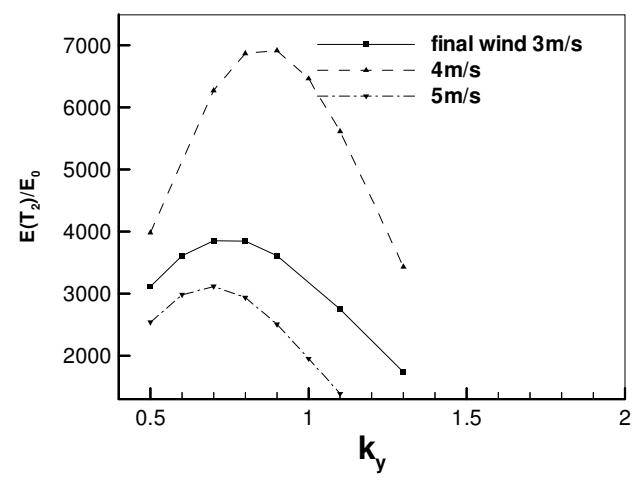

Fig. 7 Optimal energy growth for different $k_{y}$ and final wind speeds

\subsection{Dynamics of optimal initial perturbation}

In this section we consider the optimal initial perturbation and study its energy growth. For a final wind speed of $3 \mathrm{~m} / \mathrm{s}$, the optimal initial perturbation has $\lambda=2.2, k_{y}=0.7$. The perturbation amplitude and its energy growth are plotted in Fig. 8.

The optimal initial perturbation has $\hat{u}(z)=0, \hat{w}$ is obtained according to Eq. (32) and $\hat{v}$ is calculated from continuity equation (19). The energy component of $E_{v}$ and $E_{w}$ initially decays, as can be seen from Eqs. (21) and (22) that when there is no wave, no production for $\hat{v}, \hat{w}$ exists. Even shortly after $T_{1}$, which is the time wave sets in, $E_{v}$ and $E_{w}$ still decays, this is because the amplitude of wave is still small, its production is not as large as the dissipation. After longer time $E_{v}$ and $E_{w}$ begin to grow as wave amplitude become large enough to produce $\hat{v}, \hat{w}$. However, $E_{v}$ has totally different 

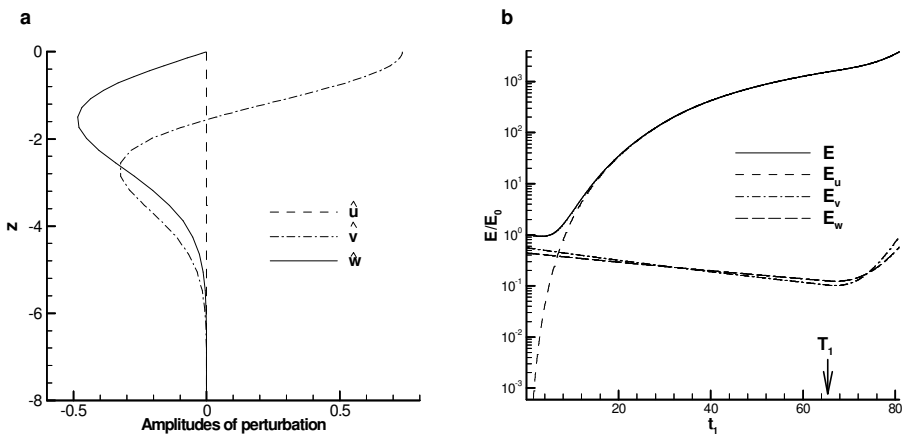

Fig. 8 (a) Amplitudes of optimal initial perturbation and (b) Energy growth for the optimal initial perturbation

behavior, it start from zero and grow quickly and become dominate after $t_{1}=10$. It continues to grow until the total energy becomes several thousands of initial energy and clear streaks are perceptible. There are two kinds of growing mechanism for $\hat{u}$. The first is lift-up effect due to $-\hat{w}_{0} \partial U_{0}^{(1)} / \partial z$ (Eq. 20), i.e., the vertical velocity lifts the current shear and produce longitudinal velocity perturbation. This process needs no wave and is an algebraic growth, or called transient growth. This growth depends on the vertical velocity. If there is no wave, as $\hat{w}$ eventually decay, the perturbation is asymptotically stable. However, if the decaying rate of $\hat{w}$ is small and the current shear $\partial U_{0}^{(1)} / \partial z$ is high enough, $\hat{u}$ is expected to become enough large to be observable. In this case, streak without wave is possible. This is possible the case of Handler et al. (2001), Tsai et al. (2001) and Lee et al. (1990), where even no surface wave is generated, streaks on the surface are indicated. However, in the case of Melville et al. (1998), the inception of Langmuir circulations always takes place after wave. When the wave interacts with the current shear, the energy grows exponentially, i.e., CL-II mechanism takes over. CL-II mechanism is supposed to become more and more important when the time becomes larger and larger. However, in the present case of Melville et al. (1998), it seems the initial preferred span-wise wave number is greatly influenced by the transient growth mechanism period, which occurs before any surface wave appears.

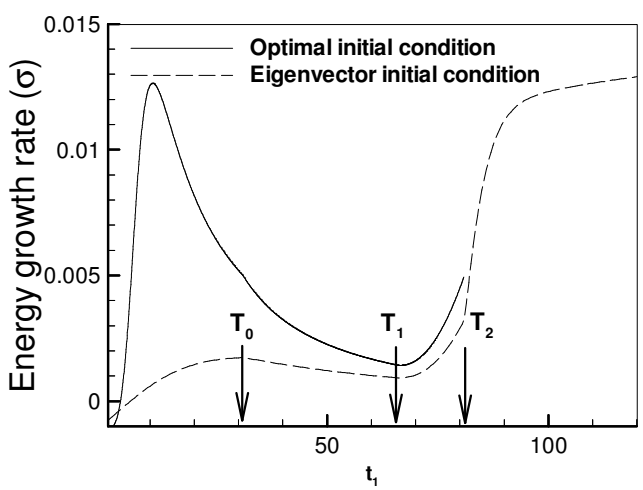

Fig. 9 Energy growth rate of perturbations with respect to time for different initial perturbations 
Fig. 9 plots the energy growth rate for optimal initial perturbation with that for eigenvector initial perturbation. The growth rate for eigenvector initial perturbation plotted here shows that after $T_{2}$ the perturbation growth rate quickly reaches almost constant 0.013 , which indicates exponential instability due to CL-II mechanism. Go back to the optimal initial perturbation, there is a significant transient growth period, the growth rate is compatible to the final exponential growth rate, say, the growth rate at $t_{1}=12$ reaches 0.0125 . As a matter of fact, the initial preferred spanwise wave number is determined by the competition of transient growth and exponential growth. It is expected that the manifested spanwise wave number becomes greater when $t_{1}>T_{2}$ as in this stage the exponential growth dominates, where the growth rate increase monotonically with spanwise wave number $k_{y}$ when $k_{y}$ is not too large.

\section{Conclusions}

The initiation of streaks in wind driving shear flow with wave addresses two processes. Firstly, the vertical gradient of longitudinal velocity (or shear) is lifted by vertical perturbation to generate longitudinal velocity perturbation. Second, wave propagate longitudinally create Stokes drift, which is able to tilt vertical vortices into longitudinal direction. The match of the above two process give birth to longitudinal vortices (Langmuir circulation), its manifestation takes the form of streaks on the surface of liquid. In this paper, transient dynamics of longitudinal vortices are studied since the initial stage of formulation of surface streaks is the purpose of study. It is found when the fully dynamics are considered, the predicted spacing of streaks is well consistent with available experimental data. The importance of initial algebraic growth is demonstrated in the formulation of streaks. The viscous effect is also included in the final determination of most unstable spanwise wave number. The present study and its results lead to the following hypothesis. The fate of any initially introduced perturbation depends on several processes. The ubiquitous viscous damping tries to calm down the perturbation. However, in all shear flows, lift-up effect produces longitudinal perturbation when there is a vertical perturbation. This does not guarantee instability as the vertical perturbation eventually decays. This process is universal in shear flow. It describe that although there is transient growth some time, shear flow is stable asymptotically. The CL-II mechanism originated from the study of Langmuir circulation here gives some hints that dramatic change takes place if there is a 2-D long-crested wave propagates along the current. Wave produces Stokes drift, small but crucial to form positive feedback loop for the generation of vertical perturbation from longitudinal perturbation, which in turns lift more and more longitudinal perturbation from basic current shear. This 2-D long-crested wave serves as a re-distributor to transform some energy from longitudinal perturbation to vertical perturbation to help to extract energy from basic shear continuously. 


\section{Acknowledgments}

This research is supported by the Hong Kong Grant Council, Project 613306 and by the Fundamental Research Funds for the Central Universities, PR China, Project DUT10LK40.

\section{References}

1. K. M. Butler, and B. F. Farrell, Phys. Fluids A, 4, 1637 (1992).

2. A. D. D. Craik, and S. Leibovich, J. Fluid Mech. 73, 401 (1976).

3. A. D. D. Craik, J. Fluid Mech. 91, 209 (1977).

4. A. D. D. Craik, J. Fluid Mech. 125, 37 (1982).

5. A. J. Faller, and E. A. Caponi, J. Geophy. Res. 83, 3617 (1978).

6. R. A. Handler, G. B. Smith and R. I. Leighton, Tellus, 53A, 233 (2001).

7. Z. Huang and C. C. Mei, J. Fluid Mech. 551, 323 (2006).

8. I. Langmuir, Science, 87, 119 (1938).

9. S. Leibovich, Ann. Rev. Fluid Mech. 15, 391 (1983).

10. S. Leibovich and S. Paolucci, J. Fluid Mech. 102, 141 (1981).

11. W. K. Melville, R. Shear, and F. Veron, J. Fluid Mech. 364, 31 (1998).

12. H. M. Nepf, and S. G. Monismith, J. Hyd. Eng. 117, 1639 (1991).

13. W. R. C. Phillips, J. Fluid Mech. 525, 215 (2005).

14. P. J. Schmid and D. S. Henningson, J. Fluid Mech. 277, 195 (1994).

15. S. A. Thorpe, Ann. Rev. Fluid Mech. 36, 55 (2004).

16. L. N.Trefethen, A. E.Trefethen, S. C. Reddy and T. A. Driscoll, Science, 261, 578 (1993).

17. W. T. Tsai, S. M. Chen and C. H. Moeng, J. Fluid Mech. 545, 163 (2005).

18. F. Veron and W. K. Melville, J. Fluid Mech. 446, 25 (2001).

19. M. Zhao, M. S. Ghidaoui and A. A. Kolyshkin, J. Fluid Mech. 570, 129 (2007).

20. M. Zhao, M. S. Ghidaoui and A. A. Kolyshkin, J. Hyd. Res. 42, 645 (2004).

21. M. Zhao, M. S. Ghidaoui and Z. Huang, On the spacing of Langmuir circulations in ocean, preparing to submit to J. Fluid Mech. (2010). 